PROCEEDINGS OF THE

AMERICAN MATHEMATICAL SOCIETY

Volume 124, Number 9, September 1996

\title{
OPERATOR VERSIONS OF THE KANTOROVICH INEQUALITY
}

\author{
P. G. SPAIN
}

(Communicated by Palle E. T. Jorgensen)

ABSTRACT. The Operator Kantorovich Inequality

$$
\left(R^{2}-r^{2}\right) u^{*}\left(a^{*} a\right) u \leq R^{2}\left(u^{*} a^{*} u\right)\left(u^{*} a u\right)
$$

holds for a wide class of operators $a$ on a Hilbert space $\mathcal{H}$ and all operators

$u: \mathcal{K} \rightarrow \mathcal{H}$ for which $[a] u$ is a partial isometry, $[a]$ being the range projection of $a$.

\section{INTRODUCTORY}

The Kantorovich Inequality

$$
4 m M\left\langle a^{-1} \xi, \xi\right\rangle \leq(m+M)^{2} \frac{\|\xi\|^{4}}{\langle a \xi, \xi\rangle},
$$

valid for a positive definite matrix with least eigenvalue $m$ and largest eigenvalue $M$, (and any nonzero vector $\xi$ ) first appeared in $[\mathrm{K}]$ and has been generalized and applied extensively since.

This inequality may be viewed as a 'reversal' of the special case

$$
\langle a \xi, \xi\rangle \leq\|a \xi\|\|\xi\|
$$

of the Cauchy-Schwarz inequality, for it is equivalent to the inequality

$$
2 \sqrt{m M}\|a \xi\|\|\xi\| \leq(m+M)\langle a \xi, \xi\rangle .
$$

The methods of operator and spectral theory allow one to generalize the inequality to a wide class of operators on Hilbert space. If $\Gamma$ is a nonzero complex number, $R=|\Gamma|$, and $0 \leq r \leq R$, then

$$
\left(R^{2}-r^{2}\right)^{\frac{1}{2}}\|a \xi\|\|[a] \xi\| \leq R|\langle a \xi, \xi\rangle|, \quad \xi \in \mathcal{H},
$$

$[a]$ being the range projection of $a$, when $a \in \mathcal{C}_{\Gamma, r}$. See below for the definition of $\mathcal{C}_{\Gamma, r}$ : it includes all $a$ for which $\sigma(a) \backslash 0$ lies in a disc of radius $r$ with centre $\Gamma$, and also the norm-ball of radius $r$ about $\Gamma 1_{\mathcal{H}}$.

This generalizes further:

$$
\left(R^{2}-r^{2}\right) u^{*}\left(a^{*} a\right) u \leq R^{2}\left(u^{*} a^{*} u\right)\left(u^{*} a u\right)
$$

for $a \in \mathcal{C}_{\Gamma, r}$ and all $u$ for which $[a] u$ is a partial isometry.

I am indebted to Dr. Shuangzhe Liu for bringing this topic to my attention.

Received by the editors March 23, 1995.

1991 Mathematics Subject Classification. Primary 47A63; Secondary 15A45, 65F65.

Key words and phrases. Kantorovich Inequality, Cauchy-Schwarz Inequality.

(C)1996 American Mathematical Society 


\section{NOTATION \& TERMINOLOGY}

Recall that when $\mathcal{H}$ is a Hilbert space a projection on $\mathcal{H}$ is an hermitian idempotent in $L(\mathcal{H})$.

A projection $e$ on $\mathcal{H}$ is a covering projection for an operator $a$ on $\mathcal{H}$ if $e a=a$. The range projection $[a]$ of $a$ is its smallest covering projection.

An operator $a \in L(\mathcal{H})$ is positive if $\langle a \xi, \xi\rangle \geq 0(\xi \in \mathcal{H})$ : equivalently, if $\sigma(a) \geq 0$, where $\sigma(a)$ is the spectrum of $a$.

Each operator $a \in L(\mathcal{H})$ has a modulus $|a|=\left(a^{*} a\right)^{\frac{1}{2}} \geq 0$ in $L(\mathcal{H})$.

Given operators $a \in L(\mathcal{H})$ and $u \in L(\mathcal{K}, \mathcal{H})$ let

$$
a^{u}=u^{*} a u \text {. }
$$

Then $|a|^{2 u}=u^{*}\left(a^{*} a\right) u$, while $\left|a^{u}\right|^{2}=\left(u^{*} a^{*} u\right)\left(u^{*} a u\right)$.

Recall that the operator $a$ is normal if $a^{*} a=a a^{*}$. If $e$ is a covering projection for a normal operator $a$, then $a e=a$. (Proof: verify that $(a e-a)^{*}(a e-a)=0$.)

Operators that are invertible on their range. If the operator $a$ is invertible on its range, that is, if the operator $\alpha=[a] a[a]=a[a]$ is invertible in $L([a] \mathcal{H})$, then $m \leq|\alpha| \leq M$ on $[a] \mathcal{H}$, where $M=\|\alpha\|, m=\left\|\alpha^{-1}\right\|^{-1}$.

A normal operator $a$ is invertible on its range if and only if either $0 \in \rho(a)$ or $0 \in \sigma(a)$ and 0 is an isolated point of $\sigma(a)$. If, further, $\sigma(a) \backslash 0$ lies in an open half-plane that does not contain 0 , then

$$
\sigma(a) \backslash 0 \subseteq \mathcal{D}
$$

for some disc $\mathcal{D}$ not containing 0 .

\section{PARTIALLY ISOMETRIC PAIRS}

Let $\mathcal{K}$ be a Hilbert space and let $u \in L(\mathcal{K}, \mathcal{H})$. Recall that the operator $u$ is a partial isometry if $u^{*} u$ is a projection: equivalently, if $u u^{*}$ is a projection.

I shall say that $(u, e)$ is a partially isometric pair if $e u\left(\right.$ or $\left.u^{*} e\right)$ is a partial isometry: equivalently, if $u^{*} e u$ (or $e u u^{*} e$ ) is a projection. (This condition has already been considered, though only in the finite-dimensional case: see [BP].)

Three sorts of partially isometric pairs are especially to be noticed: minimal, $u$-unitary and e-identity.

minimal: For $\xi \in \mathcal{H}$ define

$$
u_{\xi}: \mathbb{C} \rightarrow \mathcal{H}: \lambda \rightarrow \lambda \xi
$$

Then, identifying $\mathbb{C}$ and $L(\mathbb{C})$ canonically,

$$
u_{\xi}^{*} a u_{\xi}=\langle a \xi, \xi\rangle, \quad a \in L(\mathcal{H}) .
$$

So $\left(u_{\xi /\|e \xi\|}, e\right)$ is a partially isometric pair provided that $e \xi \neq 0$.

$u$-unitary: If $u: \mathcal{K} \rightarrow \mathcal{H}$ is unitary then $(u, e)$ is a partially isometric pair for any projection $e$.

e-identity: If $u: \mathcal{K} \rightarrow \mathcal{H}$ is a partial isometry then $\left(u, 1_{\mathcal{H}}\right)$ is a partially isometric pair.

Fundamental Lemma. Let $\mathcal{H}, \mathcal{K}$ be Hilbert spaces, let e be a projection on $\mathcal{H}$ and let $u: \mathcal{K} \rightarrow \mathcal{H}$ be such that $(u, e)$ is a partially isometric pair: i.e. suppose that $e^{u}\left(=u^{*} e u\right)$ is a projection. Then

$$
e u e^{u}=e u .
$$


The proof is simple: just multiply out to verify that $\left(e u e^{u}-e u\right)^{*}\left(e u e^{u}-e u\right)=0$.

Corollary. Let a be an operator on $\mathcal{H}$, let $e$ be a covering projection for a and let $(u, e)$ be a partially isometric pair. Then

$$
e^{u} a^{u}=a^{u} .
$$

Further, if a is normal then

$$
a^{u} e^{u}=a^{u}
$$

\section{Fundamental IDENTITY}

Let $a$ be an operator on $\mathcal{H}$, let $e$ be a covering projection for $a$, and let $(u, e)$ be a partially isometric pair.

Let $\Gamma$ be any complex number, let $R=|\Gamma|$, and let $r$ be any real number. Then, using the Corollary to the Fundamental Lemma,

$$
\begin{gathered}
\left|\left(R^{2}-r^{2}\right) e^{u}-\bar{\Gamma} a^{u}\right|^{2}=\left\{\left(R^{2}-r^{2}\right) e^{u}-\Gamma a^{* u}\right\}\left\{\left(R^{2}-r^{2}\right) e^{u}-\bar{\Gamma} a^{u}\right\} \\
=\left(R^{2}-r^{2}\right)^{2} e^{u 2}-\left(R^{2}-r^{2}\right)\left\{\bar{\Gamma} e^{u} a^{u}+\Gamma a^{* u} e^{u}\right\}+R^{2}\left|a^{u}\right|^{2} \\
=\left(R^{2}-r^{2}\right)^{2} e^{u}-\left(R^{2}-r^{2}\right)\left\{\bar{\Gamma} a^{u}+\Gamma a^{* u}\right\}+R^{2}\left|a^{u}\right|^{2},
\end{gathered}
$$

while

$$
\begin{aligned}
\left\{r^{2} e-|a-\Gamma e|^{2}\right\}^{u} & =u^{*}\left\{r^{2} e-\left(a^{*}-\bar{\Gamma} e\right)(a-\Gamma e)\right\} u \\
& =-\left(R^{2}-r^{2}\right) e^{u}-|a|^{2 u}+\bar{\Gamma} a^{u}+\Gamma a^{* u}:
\end{aligned}
$$

so

$$
\begin{aligned}
& R^{2}\left|a^{u}\right|^{2}-\left(R^{2}-r^{2}\right)|a|^{2 u} \\
& \quad \equiv\left|\left(R^{2}-r^{2}\right) e^{u}-\bar{\Gamma} a^{u}\right|^{2}+\left(R^{2}-r^{2}\right)\left\{r^{2} e-|a-\Gamma e|^{2}\right\}^{u},
\end{aligned}
$$

which is the Fundamental Identity.

THE CLASS $\mathcal{C}_{\Gamma, r}$

Let $\Gamma$ be any nonzero complex number, let $R=|\Gamma|$, and suppose that $0 \leq r \leq R$.

The class $\mathcal{C}_{\Gamma, r}$, defined by

$$
\mathcal{C}_{\Gamma, r}=\left\{a \in L(\mathcal{H}):|a-\Gamma[a]|^{2} \leq r^{2}[a]\right\}
$$

(where $[a]$ is the range projection of $a$ ), is the natural class of operators for which I can prove an Operator Kantorovich Inequality.

The class $\mathcal{C}_{\Gamma, r}$ includes

$$
\mathcal{N}_{\Gamma, r}=\left\{a \in L(\mathcal{H}): a^{*} a=a a^{*}, \quad \sigma(a) \backslash 0 \subseteq \mathcal{D}_{\Gamma, r}\right\},
$$

where

$$
\mathcal{D}_{\Gamma, r}=\{z \in \mathbb{C}:|z-\Gamma| \leq r\},
$$

and also the norm-ball

$$
\mathcal{B}_{\Gamma, r}=\left\{a \in L(\mathcal{H}):\left\|a-\Gamma 1_{\mathcal{H}}\right\| \leq r\right\} .
$$

Remark. If $a$ is normal and $a \in \mathcal{C}_{\Gamma, r}$ then $|a| \in \mathcal{C}_{|\Gamma|, r}$.

Remark. Every normal operator $a$ for which $\sigma(a) \backslash 0$ lies in an open half-plane (not containing 0 ) belongs to $\mathcal{N}_{\Gamma, r}$ for some $\Gamma$ and $r$. 
Remark. The operator with matrix $\left(\begin{array}{ll}0 & 1 \\ 0 & 0\end{array}\right)$ does not belong to $\mathcal{C}_{\Gamma, r}$ for any $\Gamma$ and $r$.

Positive Operators in $\mathcal{N}_{\Gamma, r}$. Suppose that $a \geq 0$ and that $a$ is invertible when restricted to its range: either $0 \in \rho(a)$ or if $0 \in \sigma(a)$ then 0 is an isolated point of $\sigma(a)$. Let

$$
m=\min \{\sigma(a) \backslash 0\}, \quad M=\max \sigma(a)=\|a\| .
$$

Then $a \in \mathcal{N}_{\Gamma, r}$ with $\Gamma=\frac{m+M}{2}, r=\frac{M-m}{2}$.

\section{The INEQUALITIES}

Let $\Gamma$ be any nonzero complex number, let $R=|\Gamma|$, and let $0 \leq r \leq R$.

Theorem 1 (Operator Kantorovich Inequality). Let $\mathcal{H}$ and $\mathcal{K}$ be Hilbert spaces. Suppose that $a \in \mathcal{C}_{\Gamma, r}$ and let $[a]$ be the range projection of $a$. Let $u \in L(\mathcal{K}, \mathcal{H})$ be such that $(u,[a])$ is a partially isometric pair. Then

$$
\left(R^{2}-r^{2}\right)|a|^{2 u} \leq R^{2}\left|a^{u}\right|^{2} .
$$

Proof. Immediate from the Fundamental Identity.

Corollary 1. Let a be a positive operator on $\mathcal{H}$ which is invertible on its range, let $m=\min \{\sigma(a) \backslash 0\}, M=\max \sigma(a)=\|a\|$. Let $u \in L(\mathcal{K}, \mathcal{H})$ be such that $(u,[a])$ is a partially isometric pair. Then

$$
2 \sqrt{m M}\left(a^{2 u}\right)^{\frac{1}{2}} \leq(m+M) a^{u} .
$$

This follows from Theorem 1 and from the uniqueness and monotonicity of square roots.

Corollary 2 (Scalar Version - Minimal Partially Isometric Pairs). Let $a \in \mathcal{C}_{\Gamma, r}$, and let $[a]$ be its range projection. Then

$$
\left(R^{2}-r^{2}\right)^{\frac{1}{2}}\|a \xi\|\|[a] \xi\| \leq R|\langle a \xi, \xi\rangle|, \quad \xi \in \mathcal{H} .
$$

If $a$ is positive with $\sigma(a) \backslash 0 \subseteq[m, M] \quad(0<m \leq M)$, then

$$
2 \sqrt{m M}\|a \xi\|\|[a] \xi\| \leq(m+M)\langle a \xi, \xi\rangle, \quad \xi \in \mathcal{H} .
$$

Proof. There is nothing to prove if $[a] \xi=0$ : otherwise put $u=u_{\xi /\|[a] \xi\|}$ and take square roots. The second assertion is a direct consequence of the first.

Remark. The second assertion may be proved 'in one line':

$$
\begin{aligned}
(m+ & M)^{2}\langle a \xi, \xi\rangle^{2}-4 m M\|a \xi\|^{2}\|[a] \xi\|^{2} \\
= & \left\{2 m M\|[a] \xi\|^{2}-(m+M)\langle a \xi, \xi\rangle\right\}^{2} \\
& +4 m M\langle(M-a)(a-m)[a] \xi,[a] \xi\rangle\|[a] \xi\|^{2} \\
\geq & 0 .
\end{aligned}
$$

Remark. The example $a=\operatorname{diag}\{1,-1\}$ shows that the hypothesis on the spectrum is essential. The example $a=\operatorname{diag}\{m, M\}, \quad \xi=\left(M^{\frac{1}{2}}, m^{\frac{1}{2}}\right)^{*}$ shows that the ratio $\frac{m+M}{2 \sqrt{m M}}$ may be attained so cannot be bettered.

Corollary 2 can be 'polarized': 
Corollary 3. Let $a \in \mathcal{C}_{\Gamma, r}$ and let $[a]$ be its range projection. Then

$$
\left(R^{2}-r^{2}\right)|\langle a \xi, a \eta\rangle\langle[a] \xi,[a] \eta\rangle| \leq R^{2}|\langle a \xi, \xi\rangle\langle a \eta, \eta\rangle|, \quad \xi, \eta \in \mathcal{H} .
$$

The proof:

$$
\begin{gathered}
\left(R^{2}-r^{2}\right)|\langle a \xi, a \eta\rangle\langle[a] \xi,[a] \eta\rangle| \leq\left(R^{2}-r^{2}\right)\|a \xi\|\|a \eta\|\|[a] \xi\|\|[a] \eta\| \\
=\left(R^{2}-r^{2}\right)^{\frac{1}{2}}\|a \xi\|\|[a] \xi\|\left(R^{2}-r^{2}\right)^{\frac{1}{2}}\|a \eta\|\|[a] \eta\|
\end{gathered}
$$

which, by Corollary 2,

$$
\begin{aligned}
& \leq R|\langle a \xi, \xi\rangle| R|\langle a \eta, \eta\rangle| \\
& =R^{2}|\langle a \xi, \xi\rangle\langle a \eta, \eta\rangle| .
\end{aligned}
$$

The next corollary provides an improvement of both the result and the proof of Strang $[\mathrm{S}]$.

Corollary 4. Suppose that $a$ is an operator on the Hilbert space $\mathcal{H}$ and that a is invertible on its range: i.e. the operator $\alpha=$ eae is invertible in $L(e \mathcal{H})$, where $e$ is the range projection of a. Let $M=\|\alpha\|, \quad m=\left\|\alpha^{-1}\right\|^{-1}$. Then

$$
\begin{aligned}
4 m M|\langle a e \xi, a e \eta\rangle\langle e \xi, e \eta\rangle| & \leq(m+M)^{2}\langle|\alpha| \xi, \xi\rangle\langle|\alpha| \eta, \eta\rangle, \\
4 m M\left|\langle a e \xi, e \eta\rangle\left\langle e \xi, a^{-1} e \eta\right\rangle\right| & \leq(m+M)^{2}\|e \xi\|^{2}\|e \eta\|^{2}, \quad \xi, \eta \in \mathcal{H} .
\end{aligned}
$$

Proof. I abuse notation conventionally and write $a^{-1} e \eta$ in place of $\alpha^{-1} e \eta$.

As remarked above, $m \leq|\alpha| \leq M$ on $e \mathcal{H}$. Corollary 3 applied to the operator $\alpha$ and vectors $e \xi$, e $\eta$ gives the first conclusion.

Now given $\xi, \eta \in \mathcal{H}$ there are (unique) $\theta, \phi \in e \mathcal{H}$ such that $e \xi=|\alpha|^{\frac{1}{2}} \theta, e \eta=$ $\alpha|\alpha|^{-\frac{1}{2}} \phi$. Then

$$
\begin{aligned}
\langle a e \xi, e \eta\rangle & =\langle|\alpha| \theta,|\alpha| \phi\rangle, \\
\left\langle e \xi, a^{-1} e \eta\right\rangle & =\langle\theta, \phi\rangle, \\
\|e \xi\|^{2} & =\langle|\alpha| \theta, \theta\rangle, \\
\|e \eta\|^{2} & =\langle|\alpha| \phi, \phi\rangle .
\end{aligned}
$$

Thus

$$
4 m M\left|\langle a e \xi, \eta\rangle\left\langle e \xi, a^{-1} e \eta\right\rangle\right|=4 m M|\langle|\alpha| \theta,|\alpha| \phi\rangle\langle\theta, \phi\rangle|
$$

which, by the first part of this corollary,

$$
\begin{aligned}
& \leq(m+M)^{2}\langle|\alpha| \theta, \theta\rangle\langle|\alpha| \phi, \phi\rangle \\
& =(m+M)^{2}\|e \xi\|^{2}\|e \eta\|^{2} .
\end{aligned}
$$

This immediately specialises to yield

Corollary 5 (Original Kantorovich Inequality). If $a$ is positive and invertible, with $\sigma(a) \subseteq[m, M] \quad(0<m \leq M)$, then

$$
4 m M\left\langle a^{-1} \xi, \xi\right\rangle \leq(m+M)^{2} \frac{\|\xi\|^{4}}{\langle a \xi, \xi\rangle}, \quad \xi \in \mathcal{H} .
$$


Remark. This may also be shown 'in one line':

$$
\begin{aligned}
(m+ & M)^{2}\|\xi\|^{4}-4 m M\langle a \xi, \xi\rangle\left\langle a^{-1} \xi, \xi\right\rangle \\
= & \left\{2 m M\left\langle a^{-1} \xi, \xi\right\rangle-(m+M)\|\xi\|^{2}\right\}^{2} \\
& +4 m M\left\langle(M-a)\left(1-m a^{-1}\right) \xi, \xi\right\rangle\left\langle a^{-1} \xi, \xi\right\rangle \\
\geq & 0 .
\end{aligned}
$$

\section{'DUPLEX' INEQUALITIES}

Suppose that $a$ and $b$ are operators on $\mathcal{H}$ and that $e$ is a common covering projection for $a$ and $b$ : i.e. $e a=a, e b=b$. Suppose further that $(u, e)$ is a partially isometric pair.

Let $\Gamma$ be any complex number, let $R=|\Gamma|$, and let $r$ be any real number. Then

$$
\begin{aligned}
\mid 2\left(R^{2}\right. & \left.-r^{2}\right) e^{u}-\left.\bar{\Gamma}(a+b)^{u}\right|^{2} \\
& =\left\{2\left(R^{2}-r^{2}\right) e^{u}-\Gamma(a+b)^{* u}\right\}\left\{2\left(R^{2}-r^{2}\right) e^{u}-\bar{\Gamma}(a+b)^{u}\right\} \\
& =4\left(R^{2}-r^{2}\right)^{2} e^{u}+R^{2}\left|(a+b)^{u}\right|^{2}-2\left(R^{2}-r^{2}\right)\left\{\bar{\Gamma}(a+b)^{u}+\Gamma(a+b)^{* u}\right\}
\end{aligned}
$$

while

$$
\begin{aligned}
\left\{|a-b|^{2}+2 r^{2} e-|a-\Gamma e|^{2}-|b-\Gamma e|^{2}\right\}^{u} & \\
& =-\left(a^{*} b+b^{*} a\right)^{u}-2\left(R^{2}-r^{2}\right) e^{u}+\bar{\Gamma}(a+b)^{u}+\Gamma(a+b)^{* u}:
\end{aligned}
$$

so

$$
\begin{aligned}
R^{2}\left|(a+b)^{u}\right|^{2}-2\left(R^{2}-r^{2}\right)\left(a^{*} b+b^{*} a\right)^{u} & \\
\equiv \mid & 2\left(R^{2}-r^{2}\right) e^{u}-\left.\bar{\Gamma}(a+b)^{u}\right|^{2} \\
& \quad+2\left(R^{2}-r^{2}\right)\left\{|a-b|^{2}+2 r^{2} e-|a-\Gamma e|^{2}-|b-\Gamma e|^{2}\right\}^{u}
\end{aligned}
$$

which is the Duplex Fundamental Identity. The next theorem follows from this.

Theorem 2 (Duplex Kantorovich Inequality). Suppose that $a$ and $b$ are operators on $\mathcal{H}$ with common range projection $e$ and that both $a$ and $b$ belong to the class $\mathcal{C}_{\Gamma, r}$. Suppose also that $(u, e)$ is a partially isometric pair. Then

$$
2\left(R^{2}-r^{2}\right)\left(a^{*} b+b^{*} a\right)^{u} \leq R^{2}\left|(a+b)^{u}\right|^{2} .
$$

When $a=b$ one recovers

$$
\left(R^{2}-r^{2}\right)|a|^{2 u} \leq R^{2}\left|a^{u}\right|^{2} .
$$

Theorem 3 (Positive Duplex Case). Suppose that $a$ and $b$ are both positive operators on $\mathcal{H}$, that they commute, have common range projection e, and are invertible on their range. Let

$$
\begin{array}{ll}
m_{1}=\min \{\sigma(a) \backslash 0\}, & M_{1}=\max \sigma(a), \\
m_{2}=\min \{\sigma(b) \backslash 0\}, & M_{2}=\max \sigma(b) .
\end{array}
$$

Then

$$
4\left(m_{1} M_{2}+m_{2} M_{1}\right)(a b)^{u} \leq\left|\left(m_{2} a+M_{1} b\right)^{u}\right|^{2}+\left|\left(M_{2} a+m_{1} b\right)^{u}\right|^{2}
$$


for

$$
\begin{aligned}
\mid\left(m_{2} a+\right. & \left.M_{1} b\right)\left.^{u}\right|^{2}+\left|\left(M_{2} a+m_{1} b\right)^{u}\right|^{2}-4\left(m_{1} M_{2}+m_{2} M_{1}\right)(a b)^{u} \\
= & \left|2 m_{2} M_{1} e^{u}-\left(m_{2} a+M_{1} b\right)^{u}\right|^{2}+\left|2 m_{1} M_{2} e^{u}-\left(M_{2} a+m_{1} b\right)^{u}\right|^{2} \\
& +4 m_{2} M_{1}\left\{e\left(M_{1}-a\right)\left(b-m_{2}\right) e\right\}^{u}+4 m_{1} M_{2}\left\{e\left(M_{2}-b\right)\left(a-m_{1}\right) e\right\}^{u} .
\end{aligned}
$$

This may be compared with the Generalized Pólya-Szegö Inequality of Greub \& Rheinboldt [GR], which the next theorem extends.

Theorem 4. Suppose that $a$ and $b$ are commuting normal operators, with common range projection $e$, and are invertible on their range. Let

$$
\begin{array}{ll}
m_{1}=\left\|(e a e)^{-1}\right\|^{-1}, & M_{1}=\|e a e\|, \\
m_{2}=\left\|(e b e)^{-1}\right\|^{-1}, & M_{2}=\|e b e\| .
\end{array}
$$

Then

$$
\begin{aligned}
& 4 m_{1} m_{2} M_{1} M_{2}|\langle a \xi, a \eta\rangle\langle b \xi, b \eta\rangle| \\
& \quad \leq\left(m_{1} m_{2}+M_{1} M_{2}\right)^{2}\langle|a| \xi,|b| \xi\rangle\langle|a| \eta,|b| \eta\rangle, \quad \xi, \eta \in \mathcal{H} .
\end{aligned}
$$

If $($ eae $)(\text { ebe })^{-1} \geq 0$, if, in particular, $a \geq 0, b \geq 0$, then

$$
\begin{aligned}
& 4 m_{1} m_{2} M_{1} M_{2}|\langle a \xi, a \eta\rangle\langle b \xi, b \eta\rangle| \\
& \quad \leq\left(m_{1} m_{2}+M_{1} M_{2}\right)^{2}\langle a \xi, b \xi\rangle\langle a \eta, b \eta\rangle, \quad \xi, \eta \in \mathcal{H} .
\end{aligned}
$$

Proof. Note that $\left\|(e a e)(e b e)^{-1}\right\| \leq \frac{M_{1}}{m_{2}}$, while $\left\|(e b e)(e a e)^{-1}\right\|^{-1} \geq \frac{m_{1}}{M_{2}} ;$ apply Corollary 4 to the operator $(e a e)(e b e)^{-1}$ and the vectors $b \xi$ and $b \eta$.

\section{REFERENCES}

[BP] JK Baksalary \& S Puntanen, Generalized matrix versions of the Cauchy-Schwarz and Kantorovich inequalities, Aequationes Mathematicae 41 (1991), 103-110. MR 91k:15038

[GR] W Greub \& W Rheinboldt, On a generalization of an inequality of L.V. Kantorovich, Proc American Math Soc 10 (1959), 407-415. MR 21:3774

[K] L V Kantorovich, Functional analysis and applied mathematics (Russian), Uspekhi Mat Nauk (NS) 3 (1948), 89-185. MR 10:380a

[S] W G Strang, On the Kantorovich Inequality, Proc American Math Soc 11 (1959) 468. MR 22:2904

Department of Mathematics, University of Glasgow, Glasgow G12 8QW, Scotland

E-mail address: pgs@maths.gla.ac.uk 